\title{
Hamstring Structural Injury in Futsal Players: The Effect of Active Range of Motion (AROM) Deficit on Rehabilitation Period
}

\author{
L. Smirnova ${ }^{1}$, A. Derinov리 ${ }^{2}$ I. Glazkova ${ }^{3}$ \\ 1 Department of Dermatology and Venereology, Sechenov First Moscow State Medical University, Moscow, \\ Russian Federation \\ 2 Department of Surgery, Sechenov First Moscow State Medical University, Moscow, Russian Federation \\ 3 Department of Pharmacy, Sechenov First Moscow State Medical University, Moscow, Russian Federation
}

\section{CORRESPONDING AUTHOR:}

Aleksandr Derinov

Department of surgery

Sechenov First Moscow State

Medical University

8-2 Trubetskaya Street

119991 Moscow, Russian Federation

E-mail: aleksandr.derinov@rambler.ru

DOI:

10.32098/mltj.04.2020.12

LEVEL OF EVIDENCE: 3B

\begin{abstract}
SUMMARY
Background. Hamstring muscle injuries are among the most common traumas occurring in athletes and football players. Thus, the rehabilitation time is crucial for their return to full athlete activity.

Objective. Knee active range of motion deficit after the hamstring injury in futsal players correlates with rehabilitation time. The objective is to examine cases of hamstring structural injury in futsal players and find association between the Active Range of Motion (AROM) deficit and rehabilitation time.

Methods. For this study, 200 futsal players with acute, first-time, unilateral posterior hamstring injuries were recruited, all men. All patients underwent clinical examination and ultrasonography. In 74 of 200 patients, sonography revealed no abnormalities in the musculoligamentary structures. Thereby, they were excluded from further investigation. Only 126 futsal players underwent the active range of motion testing. Injured areas were compared with the normal hamstrings in all athletes and with the control group. The AROM deficit was evaluated. The association between time to full recovery and the AROM deficit was identified. A control group (100 men) underwent examination in the same series of steps as the study group.

Results. Biceps femoris was the most commonly injured muscle, making up $80 \%$ of injuries. The musculotendinous junction, proximal and distal, was involved in $91 \%$ of injury cases. Twelve athletes had an AROM deficit of 26 to 35 degrees. For them, the average length of the recovery period was 2.5 months at its minimum. Seventy or $55.5 \%$ of athletes had an AROM deficit less than 15 degrees. These athletes felt fully recovered after 3-4 weeks of rehabilitation.

Conclusions: The active range of knee movement deficiency is an indicator that allows an objective prediction of rehabilitation time in futsal players.
\end{abstract}

\section{KEY WORDS}

Hamstrings; futsal players; knee active range of motion; ultrasound; classification; injury classification.

\section{INTRODUCTION}

Active sports, in particular football, are characterized by an increased traumatic risk. The most common type of sustained injuries occurring in sports are the muscle injuries, their frequency varying from 25 to $50 \%$ of all cases (1-5).
Almost all muscle injuries $(95 \%$ according to Hallen and Ekstrand (4)) can be divided in four groups: hamstring injury, adductor injury, quadriceps injury, and the calf muscle injury. Among the above groups, hamstring injuries are the most common type, presenting a third of all cases. Overall, the number of hamstring injuries varies depending on the 
competition. In football, for example, their frequency is 52 per 1000 players per year $(1,2,6)$.

The choice of treatment and rehabilitation program for muscle injuries depends on the severity of the injury. Studies classify muscle injuries differently depending on its location, size, and cause. Traditional muscle injury classification systems were devised by O'Donoghue (7), Ryan (8), and Askling (from van der Made et al. (9)). As is the case with other muscle injuries, hamstring strains are classified depending on the degree of damage to the muscular-tendon junction.

Ultrasound and Magnetic Resonance Imaging (MRI) can provide detailed information about the nature and extent of damage to the hamstring muscles $(10,11)$. To evaluate the hamstring muscle damage based on imaging findings, the Peetrons classification (12) for ultrasound and its modification for MRI are used (13). In order to provide clear terminology and classification of muscle injuries in sport, Mueller-Wohlfahrt et al. (14) published an important consensus statement. Their classification divides indirect muscle disorder/injury into functional muscle disorder (type 1 - overexertion-related muscle disorder, type 2 - neuromuscular muscle disorder) and structural muscle injury (type 3 - partial muscle tear, type 4 - subtotal or total tear). This classification system concerns symptoms, clinical signs, and location of damage as well as ultrasound and MRI data. Maffulli et al. (15) offered a similar grading system. Sant'Anna et al. (16) provide a review of other classification systems. The above grading frameworks failed to associate various characteristics of muscle injury with the rehabilitation time. The forecast of complete recovery from muscle injuries and return to play has significant financial and strategic implications. For this reason, it is important to optimize the diagnostic, therapeutic and rehabilitation processes. Approximately $30 \%$ of professional football players experience a re-injury within a year after the treatment (17). A premature return to full activity imposes a high risk of reoccurring injury. A solution to avoid this implication involves establishing a reliable set of injury severity assessment criteria. The size and location of the injury on MRI or ultrasound may be indicative of the time required to complete recovery (18). Clinical examination such as AROM deficiency assessment can be as useful as visualizing the rehabilitation time. Malliaropoulos et al. (19) investigated the correlation of AROM deficiency with the time to full recovery in Greek elite athletes. The authors showed that the active range of motion deficiency 48 hours after the unilateral damage to the hamstring correlates with the time to complete recovery. Furthermore, this indicator served as an objective and accurate measure. Other studies examined the effect of initial injury severity on the subsequent risk of repeated injuries
(20). According to authors, the low-grade hamstring muscle lesions inflicted a higher risk of re-injury as compared to high-grade hamstring muscle lesions. Furthermore, the Active Range of Motion (AROM) in elite Greek athletes was assessed (21). Unfortunately, the number of studies on the correlation between AROM deficiency and time to full recovery is insufficient.

The Range of Motion (ROM) variable can reflect alterations in the injured muscle flexibility. The results, however, may be inaccurate due to pain. The proper treatment of the injury, a more careful study and evidence on the most effective decisions are important to predict rehabilitation time and provide full, effective and quick return to sport activities.

The research hypothesis is that the knee active range of motion deficit after hamstring injury correlates with rehabilitation time in futsal players. The purpose of this study was to investigate cases of hamstring injury in futsal players and find association between the active ROM deficit and the rehabilitation period.

\section{METHODS}

\section{Participants and acceptance criteria}

A total of 200 male futsal players with first-time hamstring injuries were admitted into the study (age range, 18-23 years) between January 2010 and December 2016. No patients required surgical intervention. Exclusion criteria were concomitant bilateral or asynchronous hamstring strain (with chronic tendonitis); confirmed or suspected previous hamstring injury; extrinsic injury to the posterior thigh; pain at the palpation of the proximal hamstring tendon-bon junction; non-structural hamstring injury; proximal hamstring tendon tear, and grade IV injury according to Maffulli et al. (15).

The ultrasound showed no anatomical lesions in 74 patients (grade 0 according to Peetrons (12)). Thereby, only 126 futsal players were included for further research. The control group included 100 men of the same age who had never experienced a hamstring muscle injury.

Research protocol was approved by the Ministry of Sport of the Russian Federation and all athletes signed an informed consent to participate in the study. The study follows international ethical guidelines and recommendations for the clinical and field science research (22).

\section{Clinical assessment}

All athletes underwent an examination by a sport medicine doctor. A traumatologist was only involved in the most severe cases. All athletes had the following: a) local pain 
on palpation, and b) pain with resisted movements (e.g. hip extension, knee flexion). Athletes were managed with the PRICE protocol (Protect, Rest, Ice, Compression, and Elevation). Ice was applied for 15 minutes every hour for the first 6 hours after the injury and initial evaluation, and then every 3 hours. The thigh was protected and compressed using a compressive elastic bandage and was kept elevated. No motion was allowed for the first 6 hours and isometric exercises were encouraged for all the periarticular muscles of the hip and knee thereafter.

Clinical evaluation conducted 2 days after the injury included the following: a) inspection for bruising, b) ability to walk on level ground without pain, c) palpation of the hamstring with the athlete prone and knee extended (for presence or absence of tenderness), d) provocation of pain on isometric hamstring contraction, e) provocation of pain on passive movements (hip flexion with the knee extended and athletes supine), and f) AROM testing under the Askling protocol $(23,24)$. These parameters are important for both obtaining accurate data on injury severity and predicting the length of the recovery period.

Athletes enrolled in the study underwent clinical and ultrasound examinations. The study and control groups were exposed to the same AROM assessment procedures.

\section{Protocol processing}

The athlete was positioned supine with both hip and knee flexed to 90 degrees (figure $1 \mathbf{A}$ ). The unaffected leg was placed flat on the couch with the knee fully extended and maintained in this position throughout the test. The athlete was then instructed to actively extend the knee through the full available ROM until firm resistance was felt (figure $\mathbf{1}$ B). Meanwhile the hip was maintained at 90 degrees of flexion. The degree angle was measured by a double-arm 30 $\mathrm{cm}$ clear plastic inclinometer. The inclinometer was aligned along the femur with the reference point at the greater trochanter of the femur. All measurements were done in triplicate by the same physician in order to reduce examiner bias. The rehabilitation time is expressed as an interval from the trauma event to pre-injury sports activity (return to play). The difference in AROM data between the injured and uninjured leg was expressed as an AROM deficit.

\section{Rehabilitation protocol}

Injured athletes were supervised by experienced physiotherapists and traumatologists. The rehabilitation process was divided into 4 phases:

- traumatic or acute phase. Normalization of gait, involved the use of strapping and/or crutches;

- rehabilitation or strength phase. Regaining of pain-free ROM, starting with concentric training and progressing to eccentric training;

- functional phase. Application of limited loading and return to full activities under the supervision of a doctor or according to recommendations;

- full recovery phase. Return to full sport activities.

\section{Follow-up}

The athletes were followed weekly in the clinic during the rehabilitation program. The clinical follow-up period lasted until the athlete returned to pain-free full sports activity. All

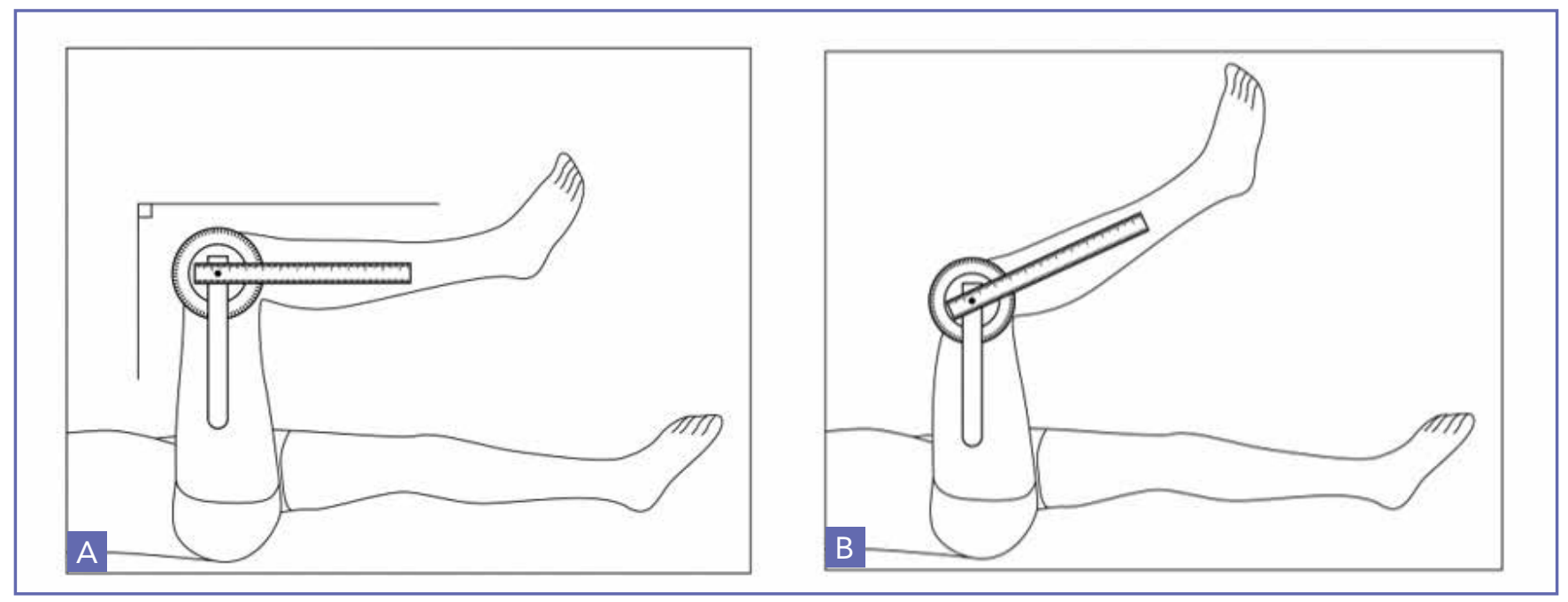

Figure 1. (A) Positioning of the inclinometer; (B) Active knee extension testing technique. 
athletes were exposed to additional remote monitoring by telephone. Telephone contacts with the athletes and their coaches were held at 3, 6, 12, 18 and 24 months after injury.

\section{Statistical analysis}

Pearson correlation was used to find correlation between the return to full athletic activity ( $4^{\text {th }}$ phase) and AROM findings. Statistical data were processed using the 1 -way analysis of variance, the Chi-Square $\left(\chi^{2}\right)$ test and regression analysis. The significance level ( $\mathrm{t}$-test) was set at $\mathrm{p}<0.05$. Statistical processing was performed with the Past v. 3.0 software.

\section{RESULTS}

Among 126 (63\%) athletes with sonographic abnormalities, 101 or $80 \%$ had injuries to the biceps femoris muscle. Musculotendinous Junction (MTJ), proximal or distal, was involved in $91 \%$ of injuries or in 115 athletes. None of the athletes had more than one injured muscle. Characteristics of muscle injuries in 126 athletes with abnormal ultrasound findings are presented in table $\mathbf{I}$.

Ultrasound scans revealed grade I injuries (according to Peetrons (12)) in almost half of the participants (62 or $49.2 \%)$, and grade II injuries in $64(50.8 \%)$ athletes.

Table I. Muscle injury characteristics in 126 athletes with abnormal findings documented by Ultrasound Imaging.

\begin{tabular}{ll}
\hline $\begin{array}{l}\text { Injured area, muscle } \\
\text { and ligaments }\end{array}$ & Number of athletes $(\%)$ \\
\hline Biceps femoris & 80.0 \\
\hline Semimembranosus & 9.0 \\
\hline Semitendinosus & 11.0 \\
\hline $\begin{array}{l}\text { Proximal musculotendinous } \\
\text { junction (MTJ) }\end{array}$ & 38.7 \\
\hline $\begin{array}{l}\text { Distal musculotendinous } \\
\text { junction (MTJ) }\end{array}$ & 52.3 \\
\hline Intramuscular tendon & 40.1 \\
\hline Myofascial injury & 9.8 \\
\hline Hematoma & 17.8 \\
\hline
\end{tabular}

The mean AROM of the injured leg in the study group was $56.12 \pm 6.9$ degrees (range, 11-90 degrees; $\mathrm{p}<0.05$ ). For asymptomatic side, it was $68.9 \pm 5.4$ degrees (range, 40-91 degrees; $\mathrm{p}<0.05)$. The mean AROM deficit in the study group was $12.8 \pm 6.8$ degrees. In the control group, the mean AROM indicated similarly to uninjured side of examined athletes, $67.9 \pm 6.5$ degrees at the range 42 to 93 degrees. There were no significant differences in AROM values between the uninjured side of the athletes and the control individuals $(\mathrm{p}<0.697)$. However, there was a significant difference $(\mathrm{p}<0.001)$ between the injured legs of athletes in the study groups and controls.

The majority of athletes in the study group (70 or $55.5 \%$ ) had an AROM deficit of less than 15 degrees. Their average recovery period ranged from three weeks to a month. Forty-four athletes, or $35 \%$, had an AROM deficit of 15 to 25 degrees. For them, this took slightly more than a month. Twelve of 126 athletes, or $9.5 \%$, experienced an AROM deficit of 25 to 35 degrees and it took them more than 2.5 months to recover (table II). The average number of days lost from futsal training was 29 days \pm 3.9 , ranging from seven up to 80 days. Hamstring strains were categorized into five grades based on the AROM deficit: grade 0 - AROM deficiency is absent, grade I - less than $15^{\circ}$, grade II $-16^{\circ}$ to $25^{\circ}$, grade III $-26^{\circ}$ to $35^{\circ}$, grade IV - over $35^{\circ}$. AROM deficit grades I and II correlate with grade I ultrasound according to Peetrons (12) $(\mathrm{r}=0.86)$, with first degree injuries according to Askling (9) $(\mathrm{r}=0.82)$, and with type $3 \mathrm{~A}$ injuries according to the comprehensive muscle injury classification $(14,15)(\mathrm{r}=0.88)$. AROM deficit grade III correlates with grade II ultrasound, second degree injuries, and type $3 \mathrm{~B}$ injuries $(\mathrm{r}=0.92, \mathrm{r}=0.84$, and $\mathrm{r}=0.85$, respectively).

\section{DISCUSSION}

The study shows a connection between AROM deficit and time to full recovery. The larger the AROM deficit, the longer the rehabilitation. With an AROM deficit of less than 15 degrees (grade I according to the present grading system), the recovery period was 3 to 4 weeks. For athletes with the AROM deficit of 15 to 25 degrees, this took slightly longer than a month. Athletes with the AROM deficit of $>25$ degrees reached full recovery by the end of

Table II. Hamstring stains in 126 athletes by clinical grade.

\begin{tabular}{lllll}
\hline Clinical grade & AROM deficit & Rehab. days & Number of athletes & $\%$ \\
\hline I & less $15^{\circ}$ & 25.9 & 70 & 55.5 \\
\hline II & $16^{\circ}$ to $25^{\circ}$ & 30.7 & 44 & 35.0 \\
\hline III & $26^{\circ}$ to $35^{\circ}$ & 75.0 & 12 & 9.5 \\
\hline
\end{tabular}


2 months. AROM deficit also correlated with the percentage of muscles involved in the injury. The results of the hamstring injury classification based on ultrasound imaging (12) largely coincided with the AROM deficit-based classification, excluding cases with the boundary percentage of muscles involved.

Clinical and ultrasound examinations were performed 48 hours after injury. In the acute setting, immediately after the injury, significant pain and disability are present. For this reason, attempts to accurately determine the athlete's ROM on the injured side would be unreliable (25).

In this study, the majority of athletes with hamstring injuries under consideration recovered their active ROM and returned to full activity in the span of 3 to 5 weeks. Rarely (1 in 10 cases), the recovery time exceeded 10 weeks. Athletes with the worst recovery prognosis can be identified early, as their ROM deficit is more than 25 degrees.

Studies using MRI (26) or ultrasound scanning $(18,24)$ showed that athletes with normal imaging returned to competition significantly faster, but there was no correlation between the presence of hematoma and the length of the rehabilitation period (18).

Other authors, however, found that fluid or hemorrhagic collections, cross-sectional involvement more than 50\%, and distal musculotendinous injury were associated with longer rehabilitation (27).

The predominance of biceps femoris injuries in the study group is consistent with other reports $(1,18,28-31)$. The incidence of semitendinosus and semimembranosus muscles in this study was $9 \%$ and $11 \%$, respectively. These indicators vary in different studies $(2,18,28,32-34)$, which suggests the existence of differences in the injury patterns between sports (35-37).

This and several other studies show that injuries occur mainly at the musculotendinous junctions (35-40). Recent studies

\section{REFERENCES}

1. McCall A, Davison M, Andersen T, et al. Injury prevention strategies at the FIFA 2014 World Cup: perceptions and practices of the physicians from the 32 participating national teams. Br J Sports Med 2015;49(9):603-608.

2. Freckleton G, Pizzari T. Risk factors for hamstring muscle strain injury in sport: a systematic review and meta-analysis. $\mathrm{Br}$ J Sports Med 2013;47:351-358.

3. Feeley BT, Kennelly S, Barnes RP, et al. Epidemiology of National Football League training camp injuries from 1998 to 2007. Am J Sports Med 2008;(8):1597-603.

4. Hallen A, Ekstrand J. Return to play following muscle injuries in professional footballers. J Sports Sci 2014;32(13):1229-36.

5. Järvinen TAH, Järvinen TLN, Kääriäinen M, Kalimo H, Järvinen M. Muscle injuries: biology and treatment. Am J Sports Med 2005;33(5):745-764. tend to differentiate injuries at the proximal and distal MTJ, as the proximal case takes more time to heal (39).

This study has several limitations. First, the research only addressed structural hamstring injuries, whereas unclear and severe cases requiring surgical treatment were excluded. The reason behind this decision is that unclear and severe situations require a different rehabilitation program. Furthermore, these cases are less informative in respect of the AROM deficit. Second, this study did not use the MRI method. Third, the study population included only the local-level futsal players and thus highlighted no injury characteristics in different sporting populations. Fourth, the athlete's behavior was unknown and could be the subject of future research.

\section{CONCLUSIONS}

This study sheds light upon the relationship between AROM deficit and time to full recovery. According the study sample, the prognosis of patients with the AROM deficit < 15 degrees was 3 to 4 weeks. Patients the AROM deficit between 15 and 25 degrees were projected to return to play in the span of one month. The average recovery time for athletes with the AROM deficit between 26 and 35 degrees was 2 months at its minimum. The AROM deficit may be considered an indicator that permits an objective prediction of time to full recovery for futsal-related hamstring injuries. Clinical evaluation has proven to be an adequate tool in recovery prognosis. Ultrasound imaging may be good for athletes having an excessive reduction in AROM, a hematoma or complete rupture of the muscle.

\section{CONFLICT OF INTERESTS}

The authors declare that they have no conflict of interests.

6. Ekstrand J, Hägglund M, Waldén M. Epidemiology of muscle injuries in professional football (soccer). Am J Sports Med 2011;39(6):1226-32.

7. O'Donoghue DO. Treatment of injuries to athletes. Philadelphia: WB Saunders, 1962.

8. Ryan AJ. Quadriceps strain, rupture and charlie horse. Med Sci Sports 1969;1:106-11.

9. van der Made AD, Wieldraaijer T, Engebretsen L, Kerkhoffs GM. Hamstring Muscle Injury. In: Kerkhoffs GM, Servien E (eds). Acute Muscle Injuries. Switzerland: Springer International Publishing, 2014:pp. 27-44.

10. Kerkhoffs GM, Van Es N, Wieldraaijer T, Sierevelt IN, Ekstrand J, van Dijk CN. Diagnosis and prognosis of acute hamstring injuries in athletes. Knee Surg Sports Traumatol Arthrosc 2013;21(2):500-9. 
11. Koulouris G, Connell D. Hamstring muscle complex: an imaging review. Radiographics 2005;25(3):571-86.

12. Peetrons P. Ultrasound of muscles. Eur Radiol 2002; 12:35-43.

13. Ekstrand J, Healy JC, Waldén M, Lee JC, English B, Hägglund M. Hamstring muscle injuries in professional football: the correlation of MRI findings with return to play. Br J Sports Med 2012;46(2):112-7.

14. Mueller-Wohlfahrt HW, Haensel L, Mithoefer K, et al. Terminology and classification of muscle injuries in sport: the Munich consensus statement. Br J Sports Med 2013;47:342-350

15. Maffulli N, Oliva F, Frizziero A, et al. ISMuLT Guidelines for muscle injuries. MLTJ 2013;3:241-249.

16. Sant'Anna JPC, de Almeida AM, Pedrinelli A, Hernandez AJ, Fernandes TL. Quality assessment of muscle injury classification in sports. MLTJ 2018;8(2):206-221.

17. Woods C, Hawkins RD, Hulse M, Hodson A. The Football Association Medical Research Programme: an audit of injuries in professional football-analysis of hamstring injuries. Br J Sports Med 2004;38:36-41.

18. Connell DA, Schneider-Kolsky ME, Hoving JL, et al. Longitudinal study comparing sonographic and MRI assessment of acute and healing hamstring injuries. Am J Roentgenol 2004;183:975-984.

19. Malliaropoulos N, Papacostas E, Kiritsi O, et al. Posterior thigh muscle injuries in elite track and field athletes. Am J Sports Med 2010;38:1813-1819.

20. Malliaropoulos N, Isinkaye T, Tsitas K, Maffulli N. Reinjury after acute posterior thigh muscle injuries in elite track and field athletes. Am J Sports Med 2011;39(2):304-10.

21. Malliaropoulos N, Kakoura L, Tsitas K, et al. Active knee range of motion assessment in elite track and field athletes: normative values. MLTJ 2015;5(3):203-7.

22. Padulo J, Oliva F, Frizziero A, Maffulli N. Muscle, Ligaments and Tendons Journal - Basic principles and recommendations in clinical and field Science Research: 2018 update. MLTJ 2018;8(3):305-307.

23. Askling CM, Tengvar M, Saartok T, Thorstensson A. Acute first-time hamstring strains during high speed running: a longitudinal study including clinical and MRI findings. Am J Sports Med 2007;35:197-206.

24. Askling CM, Tengvar M, Saartok T, Thorstensson A. Acute first-time hamstring strains during slow speed stretching: clinical, magnetic resonance imaging, and recovery characteristics. Am J Sports Med 2007;35:1716-1724.

25. Askling C, Saartok T, Thorstensson A. Type of acute hamstring strain affects flexibility, strength, and time back to pre-injury level. Br J Sports Med 2006;40:40-44.

26. Eirale C, Farooq A, Smiley FA, Tol JL, Chalabi H. Epidemiology of football injuries in Asia: a prospective study in Qatar. J Sci Med Sport 2013;16:113-117.
27. Freckleton G, Cook J, Pizzari T. The predictive validity of a single leg bridge test for hamstring injuries in australian rules football players. Br J Sports Med 2014;48:713-7.

28. De Smet AA, Best TM. MR imaging of the distribution and location of acute hamstring injuries in athletes. AJR Am J Roentgenol 2000;174(2):393-399.

29. Schut L, Wangensteen A, Maaskant J, Tol JL, Bahr R, Moen M. Can clinical evaluation predict return to sport after acute hamstring injuries? A systematic review. Sports med 2017;47(6):1123-1144.

30. Timmins R, Shield A, Williams M, Lorenzen C, Opar D. Architectural adaptations of muscle to training and injury: a narrative review outlining the contributions by fascicle length, pennation angle and muscle thickness. Br J Sports Med 2016;50(23):1467-1472.

31. Timmins RG, Bourne MN, Shield AJ, Williams MD, Lorenzen C, Opar DA. Biceps femoris architecture and strength in Athletes with a previous anterior cruciate ligament reconstruction. Med Sci Sports Exerc 2016;48:337-345.

32. Rebella G. A Prospective Study of Injury Patterns in Collegiate Pole Vaulters. Am J Sports Med 2015;43:808-815.

33. Nilstad A, Andersen TE, Bahr R, Holme I, Steffen K. Risk factors for lower extremity injuries in elite female soccer players. Am J Sports Med 2014;42:940-948.

34. Opar DA, Serpell BG. Is there a potential relationship between prior hamstring strain injury and increased risk for future anterior cruciate ligament injury? Arch Phys Med Rehabil 2014;95:401-405.

35. Zvijac JE, Toriscelli TA, Merrick S, Kiebzak GM. Isokinetic concentric quadriceps and hamstring strength variables from the NFL scouting combine are not predictive of hamstring injury in first-year professional football players. Am J Sports Med 2013;41:1511-1518.

36. Gladilina IP, Yumashev AV, Avdeeva TI, Fatkullina AA, Gafiyatullina EA. Psychological and pedagogical aspects of increasing the educational process efficiency in a university for specialists in the field of physical education and sport. Espacios 2018;39(21):11.

37. Gabbett TJ. The training-injury prevention paradox: should athletes be training smarter and harder? Br J Sports Med 2016;50:273-280.

38. Yeung SS, Suen AMY, Yeung EW. A prospective cohort study of hamstring injuries in competitive sprinters: preseason muscle imbalance as a possible risk factor. Br J Sports Med. 2009;43:589-594.

39. Bourne MN, Opar DA, Williams MD, Shield AJ. Eccentric knee flexor strength and risk of hamstring injuries in Rugby Union: a prospective study. Am J Sports Med 2015;43:2663-2670.

40. Bianchi S, Martinoli C. Ultrasound of the Musculoskeletal System. Berlin: Springer-Verlagy, 2007. 